\title{
Archivos de Cardiología de Méxíco

\section{Angioplastia primaria en diabéticos vs. no diabéticos con infarto agudo de miocardio: predictores de mortalidad}

\author{
José Alvarez ${ }^{\mathrm{a}, \mathrm{b}, *}$, Guillermo Migliaro ${ }^{\mathrm{a}, \mathrm{b}}$, Gustavo Leiva ${ }^{\mathrm{a}, \mathrm{b}}$, \\ Maria Luz Fernández-Recalde ${ }^{c}$, Brian Donato ${ }^{a, b}$, Pablo Baglioni ${ }^{\mathrm{a}, \mathrm{b}}$, \\ Alejandra Morales-Lezica ${ }^{d}$, Christian Smith ${ }^{c}$ y Jorge Allin ${ }^{a, b}$
}

\footnotetext{
a Servicio de Hemodinamia y Cardioangiología Intervencionista, Hospital Británico de Buenos Aires, Ciudad Autónoma de Buenos Aires, Argentina

b Servicio de Hemodinamia y Cardioangiología Intervencionista, Hospital Alemán , Ciudad Autónoma de Buenos Aires, Argentina

c Servicio de Cardiología, Hospital Británico de Buenos Aires, Ciudad Autónoma de Buenos Aires, Argentina

d Servicio de Cardiología, Hospital Alemán , Ciudad Autónoma de Buenos Aires, Argentina
}

Recibido el 22 de diciembre de 2014; aceptado el 27 de agosto de 2015

\section{PALABRAS CLAVE \\ Diabetes mellitus; \\ Infarto agudo de \\ miocardio; \\ Angioplastia \\ primaria; \\ Argentina}

\begin{abstract}
Resumen
Introducción y objetivos: La diabetes es una enfermedad con elevado riesgo cardiovascular. El objetivo fue evaluar la mortalidad intrahospitalaria y en el seguimiento a largo plazo de los pacientes diabéticos con infarto agudo de miocardio tratados con angioplastia primaria y determinar sus predictores.

Material y métodos: Se incluyeron de forma retrospectiva 866 pacientes durante enero de 1993 y diciembre de 2013. Se evaluaron 100 sujetos con diagnóstico de diabetes. La mediana de seguimiento fue de 121 meses.

Resultados: De los 100 pacientes diabéticos evaluados (11.56\% del total), el $86 \%$ fueron hombres y el 50\% tenían más de 50 años. El 76\% estaba en Killip-Kimball 1 al ingreso y el 16\% en KillipKimball 4. La localización más frecuente fue la anterior y el 65\% presentaba lesión de 2 o más vasos coronarios.

Se observó una mortalidad intrahospitalaria del 15\%, cuyo único predictor resultó el KillipKimball de ingreso.

La mortalidad alejada fue del 35\%, y solamente la edad, el Killip-Kimball al ingreso y el uso de Inhibidores de la enzima convertidora de angiotensina resultaron variables con significación estadística. A diferencia de la población diabética, el Killip-Kimball al ingreso no se asoció significativamente a la mortalidad alejada en no diabéticos.
\end{abstract}

\footnotetext{
* Autor para correspondencia. Perdriel 74 CABA (C1280AEB). Argentina. Fax: +54 11 43096400, int. 2361. Correo electrónico: jagalvarez@intramed.net (J. Alvarez).
} 
Conclusión: Esta población de pacientes diabéticos se caracterizó por ser más añosa y por presentar lesión de 2 o más vasos coronarios. El shock cardiogénico fue la única variable predictora independiente de mortalidad intrahospitalaria y, junto con la edad y el uso de inhibidores de la enzima convertidora de angiotensina, de mortalidad en el seguimiento a largo plazo. (c) 2015 Instituto Nacional de Cardiología Ignacio Chávez. Publicado por Masson Doyma México S.A. Todos los derechos reservados.

\section{KEYWORDS}

Diabetes mellitus ;Acute Myocardial Infarction; Primary Angioplasty; Argentina

\section{Primary angioplasty in diabetic and non-diabetic patients with acute myocardial infarction: Predictors of mortality}

\begin{abstract}
Background and aims: Diabetes mellitus is one of the major risk factors for coronary artery disease. The aim of this study was to evaluate in-hospital mortality and during follow-up of diabetic patients with acute myocardial infarction treated with primary angioplasty and to determine its predictors.

Materials and methods: Eight hundred and sixty six patients were retrospectively enrolled from January 1993 to December 2013. A hundred patients with a diagnosis of diabetes were evaluated. The median follow-up was 121 months in $90 \%$ of the population.

Results: Of the 100 diabetic patients included (11.56\%) $86 \%$ were male and $50 \%$ older than 70 years. Overall, $76 \%$ presented with a Killip-Kimball grade of 1 at admission and $16 \%$ presented with a Killip-Kimball 4. The most frequent location of myocardial infarction was anterior and $65 \%$ had 2 or more coronary vessel disease.

In-hospital mortality was $15 \%$. The only independent variable significantly associated was the Killip-Kimball at admission.

Mortality during follow up was 35\% and its independent predictors were: age, Killip-Kimball at admission and use of angiotensin-converting enzyme inhibitors Interestingly, in the non-diabetic group, Killip-kimball at admission failed to predict long-term mortality

Conclusion: This group of diabetic patients was older, and with a higher prevalence of 2 or more vessel disease. Cardiogenic shock on admission was the only independent predictor of in-hospital death and along with age and angiotensin-converting enzyme inhibitor use, an independent predictor of mortality during long term follow-up.

(C) 2015 Instituto Nacional de Cardiología Ignacio Chávez. Published by Masson Doyma México S.A. All rights reserved.
\end{abstract}

\section{Introducción}

La diabetes mellitus (DM) es una enfermedad metabólica vinculada a un riesgo significativo de enfermedad microy macrovascular ${ }^{1,2}$ que está presente en el $20-30 \%$ de los pacientes con síndromes coronarios agudos y en el 15$20 \%$ de aquellos que requieren algún procedimiento de revascularización ${ }^{3}$.

Se describe en estos pacientes un estado hipercoagulable con mayor actividad plaquetaria y mayor capacidad de generación de fibrina, y una arteriopatía con placas con mayor contenido de macrófagos, enfermedad más difusa y calcificada, y escaso fenómeno de dilatación compensadora ${ }^{4}$.

Los pacientes con DM que presentan un síndrome coronario agudo con o sin supradesnivel ST tienen mayor riesgo de mortalidad a corto y largo plazo, así como mayor prevalencia de reinfarto e insuficiencia cardíaca ${ }^{5}$.

En pacientes diabéticos con infarto agudo de miocardio con elevación del ST (IAMCST) la angioplastia primaria (AP) es el tratamiento de elección ${ }^{6,7}$.

Evidencia previamente publicada ha demostrado que, en este contexto, la mortalidad inmediata y alejada es mayor que en sujetos no diabéticos ${ }^{8-10}$.
Poca evidencia evalúa aquellos predictores de mortalidad a corto y largo plazo en la población de pacientes diabéticos con IAMCST sometidos a AP en particular.

\section{Materiales y métodos}

\section{Diseño del estudio y selección de pacientes}

Se diseñó un estudio de tipo corte transversal retrospectivo y multicéntrico. El mismo fue llevado a cabo en 2 centros hospitalarios de la ciudad de Buenos Aires. Se recopilaron los datos de todos los pacientes con IAMCST sometidos a AP entre enero de 1993 y diciembre de 2013. Se utilizaron las bases de datos de los servicios de cardiología intervencionista de ambos centros, que son periódicamente actualizadas en forma prospectiva con los datos de seguimiento de los pacientes intervenidos. Aquellos datos faltantes fueron recolectados por medio de la búsqueda en las historias clínicas o a través de comunicación telefónica con el paciente.

Se incluyeron aquellos pacientes con diagnóstico de IAMCST tratados con AP. Se definieron los grupos primario 
y secundario de estudio con relación a si presentaban o no DM.

Se definió como pacientes con DM a aquellos sujetos con diagnóstico previo en tratamiento con hipoglucemiantes o antihiperglucemiantes orales o insulina. EL IAMCST fue definido por la presencia de síntomas de isquemia miocárdica con menos de $12 \mathrm{~h}$ de evolución asociado a elevación del segmento ST en el punto $\mathrm{J}$ del electrocardiograma $\geq$ de $2 \mathrm{~mm}$ en por lo menos 2 derivaciones contiguas o la presencia de nuevo o presuntamente nuevo bloqueo completo de la rama izquierda más liberación de biomarcadores de necrosis miocárdica.

Fueron excluidos aquellos sujetos con infarto agudo de miocardio de evolución superior a $12 \mathrm{~h}$, o aquellos que habían recibido tratamiento previo con fibrinolíticos, ya sea con o sin criterios de reperfusion.

\section{Descripción del procedimiento}

La angioplastia fue realizada a través de abordaje de las arterias femoral o radial con catéteres de 6 o 7 french. La anticoagulación se llevó a cabo con heparina intravenosa en dosis ajustadas para mantener un tiempo de coagulación activado mayor a $250 \mathrm{seg}$; en los casos en que se planeó la utilización de abciximab la dosis inicial de heparina se redujo a $70 \mathrm{UI} / \mathrm{kg}$ de peso. Guías, balones y catéteres de tromboaspiración se utilizaron según el criterio del operador. Los stents fueron impactados a alta presión según técnica; y a partir del año 2006 se utilizaron stents recubiertos siempre que estuvieron disponibles. La duración de la doble antiagregación plaquetaria fue a criterio del médico de cabecera con una fuerte recomendación de mantenerla durante 12 meses en los casos de utilización de stents recubiertos.

\section{Variables evaluadas en seguimiento}

Fueron evaluadas las siguientes variables relativas a la internación: edad, sexo, antecedentes de infarto previo, antecedentes de tabaquismo, dislipidemia, hipertensión arterial, cirugía de revascularización miocárdica previa, angioplastia previa, grado de Killip-Kimball (KK) al ingreso, localización del infarto, vaso responsable del evento, número de vasos con enfermedad aterosclerótica significativa (estenosis $\geq 70 \%$ ), score de Syntax, colocación de stent, uso de catéter de tromboaspiración, administración de inhibidores de la glucoproteína ॥b/ıla, flujo TIMI postangioplastia y mortalidad intrahospitalaria $(\mathrm{MIH})$ periprocedimiento. Luego del egreso hospitalario, se realizaron los controles clínicos periódicos para determinar las siguientes variables en seguimiento, que conformaron nuestro objetivo primario: muerte en el seguimiento alejado (MS), reinfarto, requerimiento de cirugía de revascularización miocárdica, nueva angioplastia y accidente cerebrovascular.

Como objetivos secundarios, se compararon las MIH y MS de los sujetos diabéticos incluidos con respecto a aquellas de los pacientes no diabéticos de nuestra cohorte. De la misma forma, se estimaron las variables independientes asociadas a MIH y MS en sujetos no diabéticos para contrastarlas con los hallazgos en el grupo de diabéticos.

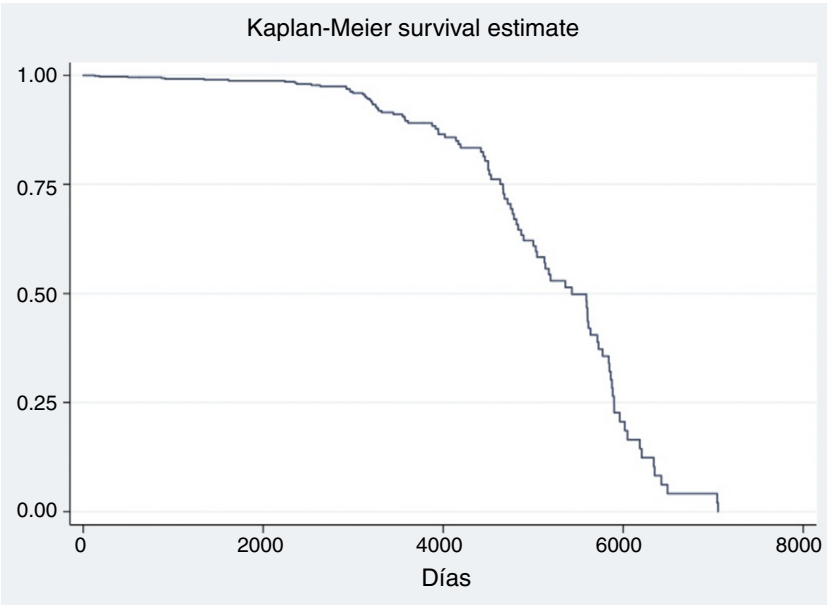

Figura 1 Mortalidad en seguimiento global en la población estudiada.

\section{Análisis estadístico}

A partir de las bases de datos analizadas, se realizó el análisis estadístico utilizando el programa Stata (Version 11.2, Statacorp, College Station, Texas, EE. UU.). Se describieron las variables categóricas como porcentajes y las variables numéricas como media con su desviación estándar o mediana con su rango intercuartílico 25-75\%, según correspondiera. Se calcularon los odds ratio (OR) con su respectivos intervalos de confianza del 95\%.

Las medidas de efecto evaluadas fueron: MIH y MS. Aquellas variables significativamente asociadas con las medidas antedichas fueron incluidas en un análisis multivariado siguiendo un modelo de regresión logística. Se consideró como significativo un valor de $p<0.05 \%$. Se confeccionó una curva de tipo Kaplan-Meier con los datos obtenidos de mortalidad en seguimiento tanto para la población en general como para la comparación entre diabéticos y no diabéticos.

\section{Resultados}

En el período de estudio se encontraron un total de 866 pacientes sometidos a AP. De ellos, 100 sujetos correspondieron al grupo de pacientes con DM. La tabla 1 describe las características generales de los pacientes incluidos. La edad promedio fue de $65 \pm 10$ años, en su mayoría correspondieron al sexo masculino (86\%), tuvieron mayor incidencia de hipertensión arterial, peor estado hemodinámico al ingreso (KK III y IV) y en su tratamiento hubo mayor utilización de inhibidores glucoproteicos ॥b/ıla. De los pacientes que sobrevivieron al evento, el $10 \%$ fueron perdidos en seguimiento de largo plazo cuya mediana fue de 121 (109-145) meses.

Se observó una MIH en diabéticos del 15\%, mientras que la MS fue del $35 \%$. (figs. 1 y 2). Esto contrasta con la mortalidad de los sujetos no diabéticos de nuestra cohorte (MIH 4.69\%, OR 3.76 [1.95-7.23]; MS 12.75\%, OR 2.79 [1.56-4.98]), tal como se observa en la figura 3. Excluyendo a aquellos sujetos con KK 4 al ingreso, la MIH fue del $2 \%$ y la MS del $21 \%$. 
Tabla 1 Características generales y demográficas de la población

\begin{tabular}{|c|c|c|c|}
\hline Variable & Diabetes \% (n/N) & No diabetes \% (n/N) & $\mathrm{p}$ \\
\hline $\operatorname{Sexo}(F)$ & $14(14 / 100)$ & $24(184 / 766)$ & 0.87 \\
\hline Edad: menor 70 años & $50(50 / 100)$ & $75(574 / 766)$ & 0.0001 \\
\hline Dislipidemia & $48(48 / 100)$ & $45(345 / 766)$ & 0.20 \\
\hline Tabaquismo & $42(42 / 100)$ & $52(398 / 766)$ & 0.67 \\
\hline Hipertensión arterial & $66(66 / 100)$ & $45(345 / 766)$ & 0.03 \\
\hline IAM previo & $20(20 / 100)$ & $9.5(73 / 766)$ & 0.06 \\
\hline Killip-Kimball ingreso & $76(76 / 100)$ & $84(643 / 766)$ & 0.0001 \\
\hline 1 & $5(5 / 100)$ & $9(69 / 766)$ & 0.03 \\
\hline 2 & $3(3 / 100)$ & $1(8 / 766)$ & 0.24 \\
\hline 3 & $16(16 / 100)$ & $6(46 / 766)$ & 0.03 \\
\hline 4 & & & 0.003 \\
\hline Número de vasos & $35(35 / 100)$ & $53(406 / 766)$ & 0.03 \\
\hline 1 & $65(65 / 100)$ & $47(360 / 766)$ & 0.003 \\
\hline$\geq 2$ & & & 0.003 \\
\hline Vaso responsable & $52(52 / 100)$ & $49(375 / 766)$ & 0.09 \\
\hline DA & $31(31 / 100)$ & $33(253 / 766)$ & 0.87 \\
\hline$C D$ & $16(16 / 100)$ & $16(123 / 766)$ & 0.73 \\
\hline$C X$ & $1(1 / 100)$ & $0.66(5 / 766)$ & 0.84 \\
\hline $\mathrm{TCl}$ & 0 & $1.34(10 / 766)$ & 1 \\
\hline By-pass & & & 1 \\
\hline Stent & $82(82 / 100)$ & $86(659 / 766)$ & 0.16 \\
\hline Inhibidores glucoproteicos ॥b/ıa & $39(39 / 100)$ & $31.5(241 / 766)$ & 0.02 \\
\hline Tiempo $\mathrm{pb}<120 \mathrm{~min}$ & $68.75(66 / 96)$ & $64.76(489 / 755)$ & 0.62 \\
\hline IECA en seguimiento & $71.93(41 / 96)$ & $64.55(295 / 755)$ & 0.27 \\
\hline Estatinas en seguimiento & $49(47 / 96)$ & $52(392 / 755)$ & 0.5 \\
\hline DAPT a 12 meses & $56(54 / 96)$ & $49(370 / 755)$ & 0.08 \\
\hline Ácido acetilsalicílico en seguimiento & $95(91 / 96)$ & $91(690 / 755)$ & 0.3 \\
\hline
\end{tabular}

CD: coronaria derecha; CX circunfleja; DA: arteria descendente anterior; DAPT: doble tratamiento antiplaquetario; F: femenino; IAM: infarto agudo de miocardio; IECA: inhibidores de la enzima convertidora de angiotensina; TCI: tronco de coronaria izquierda.

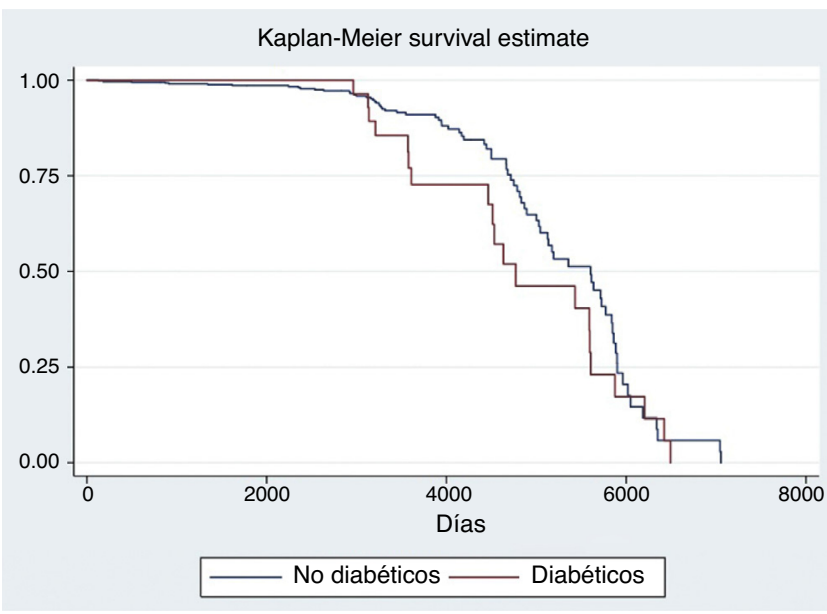

Figura 2 Mortalidad en seguimiento; comparativa entre diabéticos y no diabéticos.

En el seguimiento a largo plazo, la prevalencia de eventos cardiovasculares mayores en sujetos diabéticos fue del $32 \%$; de ellos, el 69\% requirieron nueva angioplastia. En contraposición, la prevalencia de MACE en sujetos no diabéticos fue del $5.69 \%(p<0.001)$, con un requerimiento de nueva angioplastia del $22.66 \%$.
Tabla 2 Mortalidad intrahospitalaria en diabéticos

\begin{tabular}{llc}
\hline Variable & $\begin{array}{l}\text { Análisis } \\
\text { univariado OR } \\
\text { IC 95\%) }\end{array}$ & $\begin{array}{l}\text { Análisis } \\
\text { multivariado } \\
\text { OR [(IC 95\%)] }\end{array}$ \\
\hline Sexo & $0.32(0.17-0.58)$ & $0.60(0.24-1.5)$ \\
Edad & NA & $0.91(0.8-1.05)$ \\
KK ingreso & $6.76(4.18-10.94)$ & $13.64(2.63-70.65)$ \\
TIMI final & $0.38(0.17-0.83)$ & $0.29(0.04-2.06)$ \\
Score de Syntax & 2.66 (0.65-10.86) & $0.57(0.04-7.05)$ \\
\hline IC: intervalo de confianza; KK: Killip-Kimball; NA: no aplicable; \\
OR: odds ratio; TIMI: Thrombolysis In Myocardial Infarction.
\end{tabular}

\section{Predictores de mortalidad en pacientes con diabetes mellitus}

La tabla 2 muestra los análisis uni- y multivariado de los predictores de MIH. En el primero, el KK al ingreso, el flujo TIMI postangioplastia y la edad demostraron estar significativamente asociados al evento analizado. Sin embargo, en el análisis multivariado, solo el KK demostró ser factor predictor independiente asociado a MIH (OR 13.64 [2.63-70.65]).

En cuanto a la MS, la tabla 3 describe los resultados de los análisis uni- y multivariado. Se identificaron como variables significativamente asociadas en el análisis univariado a la edad, el infarto previo, el KK al ingreso, el número de vasos 


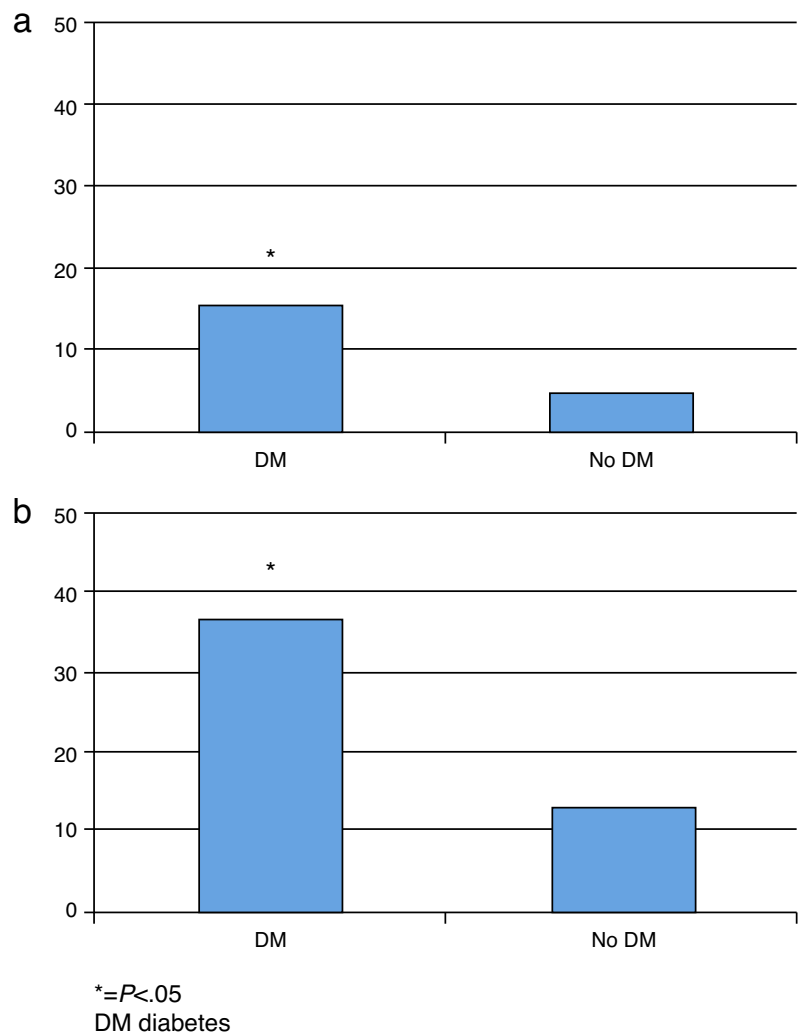

Figura 3 Mortalidad intrahospitalaria (a) y alejada (b) en seguimiento en sujetos diabéticos y no diabéticos.

comprometidos, el score de Syntax y el uso de inhibidores de la enzima convertidora de angiotensina (IECA). En el análisis multivariado, solamente la edad (OR 1.07 [1-1.16]), el KK (OR 2.48 [1.48-4.14]) y el uso de IECA (OR 0.07 [0.01-0.65]) demostraron ser factores independientes asociados con MS.

\section{Predictores de mortalidad en pacientes sin diabetes mellitus}

La tabla 4 resume los resultados del análisis uni- y multivariado relacionados con los predictores de MIH en pacientes

Tabla 3 Mortalidad en el seguimiento en diabéticos

\begin{tabular}{lll}
\hline Variable & $\begin{array}{l}\text { Análisis } \\
\text { univariado OR } \\
\text { (IC 95\%) }\end{array}$ & $\begin{array}{l}\text { Análisis } \\
\text { multivariado } \\
\text { OR (IC 95\%) }\end{array}$ \\
\hline Edad & NA & $1.07(0.99-1.17)$ \\
Menos 70 años & $0.36(0.14-0.92)$ & $1.89(0.31-11.68)$ \\
IAM previo & $2.81(1-7.93)$ & $1.81(0.52-6.28)$ \\
KK ingreso & $2.39(1.66-3.44)$ & $2.47(1.48-4.14)$ \\
Número de vasos & $2.2(1.30-3.70)$ & $1.91(0.94-3.92)$ \\
Stent & $0.35(0.12-1.04)$ & $0.42(0.11-1.61)$ \\
Score de Syntax & $4.3(1.01-18.24)$ & $2.35(0.55-10)$ \\
Uso de IECA & $0.15(0.02-0.96)$ & $0.07(0.01-0.65)$ \\
\hline
\end{tabular}

IAM: infarto agudo de miocardio; IECA: inhibidores de la enzima convertidora de angiotensina; KK: Killip-Kimball; IC: intervalo de confianza; NA: no aplicable; OR: odds ratio.
Tabla 4 Mortalidad intrahospitalaria en no-DM

\begin{tabular}{lll}
\hline Variable & $\begin{array}{l}\text { Análisis } \\
\text { univariado OR } \\
\text { (IC95\%) }\end{array}$ & $\begin{array}{l}\text { Análisis } \\
\text { multivariado } \\
\text { OR (IC95\%) }\end{array}$ \\
\hline KK ingreso & $4.87(1.68-14.11)$ & $4.45(3.06-6.46)$ \\
Tabaquismo & $0.46(0.22-0.95)$ & $0.67(0.23-1.91)$ \\
Sexo & $0.21(0.11-0.44)$ & $0.78(0.28-2.17)$ \\
Edad & NA & $1.04(0.97-1.12)$ \\
\hline
\end{tabular}

DM: diabetes mellitus; KK: Killip-Kimball; IC: intervalo de confianza; NA: no aplicable; OR: odds ratio.

Tabla 5 Mortalidad en seguimiento en no-DM

\begin{tabular}{lll}
\hline Variable & $\begin{array}{l}\text { Análisis } \\
\text { univariado OR } \\
\text { (IC95\%) }\end{array}$ & $\begin{array}{l}\text { Análisis } \\
\text { multivariado } \\
\text { OR (IC95\%) }\end{array}$ \\
\hline Edad & NA & $1.10(1.06-1.15)$ \\
Angor previo & $2.15(0.94-4.94)$ & $2.95(0.91-9.56)$ \\
KK ingreso & $4.87(1.67-14.25)$ & $1.21(0.55-2.64)$ \\
Colocación stent & $0.30(0.17-0.53)$ & $0.81(0.37-4.46)$ \\
$\begin{array}{l}\text { Uso de ácido } \\
\quad \text { acetilsalicílico }\end{array}$ & $0.15(0.06-0.37)$ & $0.64(0.11-3.53)$ \\
$\begin{array}{l}\text { Uso de betablo- } \\
\quad \text { queantes }\end{array}$ & $0.21(0.09-0.50)$ & $0.60(0.12-3.03)$ \\
$\begin{array}{l}\text { Uso de estatinas } \\
\text { Uso de IECA }\end{array}$ & $0.22(0.10-0.49)$ & $0.67(0.18-2.44)$ \\
\hline DM: & $0.23(0.10-0.52)$ & $0.27(0.10-0.69)$
\end{tabular}

DM: diabetes mellitus; IECA: inhibidores de la enzima convertidora de angiotensina; KK: Killip-Kimball; IC: intervalo de confianza; NA: no aplicable; OR: odds ratio.

no diabéticos. Coincidentemente, solo el $\mathrm{KK}$ al ingreso resultó ser el único factor independiente significativamente asociado a MIH (OR 4.45 [3.06-6.46]).

En cuanto a la mortalidad en el seguimiento (tabla 5), llama la atención que, si bien la edad (OR 1.10 [1.06-1.15]) y el uso de IECA (OR 0.27 [0.10-0.69]) presentaron una asociación significativa en relación al evento, el KK no se asoció en el análisis multivariado a la mortalidad en seguimiento (OR 1.21 [0.55-2.64]).

\section{Discusión}

En esta serie de pacientes consecutivos con IAMcST que se presentaron a la sala de cateterismos para ser tratados con $\mathrm{AP}$, en comparación con la población de pacientes sin DM, los diabéticos fueron en promedio más añosos y con mayor prevalencia de enfermedad de 2 o más vasos coronarios.

La presencia de DM se asoció a mayor mortalidad tanto en la evolución intrahospitalaria como en el seguimiento alejado. El grado de KK demostró ser un predictor consistente de MIH y MS mientras que la edad y la falta de tratamiento con IECA fueron predictores independientes de MS.

Es interesante la comparación indirecta de los predictores de riesgo de mortalidad dentro de una misma población entre diabéticos y no diabéticos. Como muestran nuestros resultados, no existen diferencias en el comportamiento de estos factores de riesgo con excepción del $\mathrm{KK}$ al ingreso que, en la población no diabética, pierde la fuerza de asociación con la MS. 
Es bien sabido que la DM presenta un riesgo incrementado de cardiopatía isquémica con enfermedad de múltiples $\operatorname{vasos}^{11,12}$. De esto se desprende la necesidad de angioplastia en el contexto de IAMCST o de angina inestable en esta población ${ }^{13}$. En este sentido, la DM cobra relevancia como factor de mal pronóstico, dada la evidencia existente que demuestra que aquellos sujetos diabéticos presentan una mayor probabilidad de muerte posterior a un evento coronario agudo a corto y largo plazo ${ }^{5,14}$. Timmer et al. ${ }^{15}$, en un estudio de seguimiento de pacientes con IAMCST aleatorizados a tratamiento fibrinolítico intravenoso con estreptocinasa frente a AP, encontraron que la DM era una variable independiente asociada a mortalidad (HR 2.3, $\mathrm{p}<0.001$ ) y que la AP se encontraba asociada a un mejor pronóstico en la población diabética en particular.

La presencia de hiperglucemia (incluso la hiperglucemia por estrés) está asociada a un mayor tamaño de infarto y a mayor mortalidad en pacientes con IAMcST. De hecho, modelos in vitro e in vivo demuestran que la hiperglucemia está involucrada en la lesión por reperfusion ${ }^{16,17}$.

En un trabajo recientemente publicado por De Luca et al. ${ }^{8}$, se evaluaron 6,298 pacientes con infarto de miocardio sometidos a AP de los cuales el $15.4 \%$ eran diabéticos. En el seguimiento alejado, los sujetos con DM presentaron mayor mortalidad (19.1 vs. $7.4 \%, p<0.0001)$, reinfarto (10.4 vs. $7.5 \%, p<0.001)$ y trombosis del stent $(7.6$ vs. $4.8 \%$, $p=0.002$ ). Jensen et al. ${ }^{3}$ describen similares resultados en cuanto a mortalidad y riesgo de reinfarto en un estudio poblacional llevado a cabo en Dinamarca, si bien en este caso la frecuencia de trombosis de stent fue similar con respecto a no diabéticos en el seguimiento (1.6 vs. $1.5 \%$, HR 1.15, IC95\% 0.5-2.67).

Sin embargo, existe poca evidencia que oriente acerca de los posibles factores que puedan asociarse a mayor mortalidad dentro de esta población en particular. Kahn et al. ${ }^{18}$ han descripto que la edad, el sexo, el score TIMI posperfusión, el tiempo puerta-balón, infarto previo y el número de vasos comprometidos fueron variables asociadas a la mortalidad en DM e IAMCST en seguimiento. De acuerdo a nuestros resultados, el deterioro hemodinámico al ingreso, evaluado por la clasificación de KK, fue la única variable predictora de mortalidad, tanto durante la internación como en el seguimiento alejado. Es bien sabido el valor pronóstico que el $\mathrm{KK}$, en particular el desarrollo de shock cardiogénico, tiene en sujetos con eventos coronarios agudos ${ }^{19}$. El pronóstico adverso del shock cardiogénico en pacientes con IAMCST y DM ha sido previamente descripto en el trabajo publicado por Gasior et al. ${ }^{20}$. De todas maneras, el KK se comporta más bien como un predictor de mortalidad a corto plazo en este contexto, existiendo menos evidencia acerca del valor del mismo con relación a la mortalidad a largo plazo.

Llama la atención que variables como el flujo TIMI postangioplastia, el uso de stents, el score de Syntax o el uso de inhibidores de la glucoproteína ॥b/ıla no demostraron estar vinculadas en forma independiente con la mortalidad de nuestra cohorte de pacientes diabéticos.

Cabe mencionar la baja compliance al tratamiento fundamentalmente con estatinas durante el seguimiento alejado de estos pacientes. Las causas del cese del tratamiento más descriptas fueron la presencia de efectos adversos al fármaco y la decisión del paciente a pesar de la prescripción médica.
La discontinuación o el no uso de IECA se asoció a una mayor MS; a diferencia de otras medicaciones, el uso de IECA a largo plazo demostró tener un efecto protector. Este hallazgo está en consonancia con estudios previos ${ }^{21}$. No se cuenta, sin embargo, con información importante para evaluar en detalle dicho impacto, como por ejemplo la dosis recibida por los pacientes que consumían IECA en el seguimiento.

La baja adherencia a fármacos en pacientes con enfermedades crónicas y su impacto sobre la mortalidad luego del egreso hospitalario en pacientes con síndromes coronarios agudos ha sido previamente descripto ${ }^{22-25}$.

Este estudio cuenta con algunas falencias que merecen ser señaladas. Por un lado, el estudio presenta las limitaciones derivadas de su naturaleza retrospectiva. Sin embargo, la base de datos utilizada en los centros intervinientes se actualiza periódicamente en forma prospectiva. Por otro lado, contamos con un tamaño muestral relativamente pequeño; es posible que ciertas variables puedan estar significativamente asociadas a mortalidad y que no hayan podido ser discriminadas dado el diseño del estudio. Asimismo, debido al tipo de estudio, al número de pacientes diabéticos ingresados y a los puntos finales estudiados no se analizaron resultados en relación con el tipo de stent utilizado. De la misma manera, carecemos de datos relevantes, como el control metabólico de los pacientes diabéticos en el seguimiento o al ingreso hospitalario y la duración de la terapia dual con ácido acetilsalicílico-clopidogrel. En buena medida, las fortalezas de este estudio provienen de su naturaleza multicéntrica y de un porcentaje de pérdida de seguimiento a largo plazo baja. Según nuestro conocimiento, este es el primer trabajo de esta naturaleza realizado en la Argentina.

\section{Conclusión}

En esta población de pacientes consecutivos con IAMcST tratados con AP la presencia de DM estuvo asociada a mayor MIH y MS. Los predictores independientes de estos eventos fueron el grado de deterioro hemodinámico al ingreso (KK) para la evolución intrahospitalaria y alejada, y la edad y el tratamiento con IECA para la mortalidad alejada.

Es necesaria una mayor evidencia con el fin de confirmar estos hallazgos y poder identificar otros predictores de mala evolución en esta población.

\section{Responsabilidades éticas}

Protección de personas y animales. Los autores declaran que para esta investigación no se han realizado experimentos en seres humanos ni en animales.

Confidencialidad de los datos. Los autores declaran que han seguido los protocolos de su centro de trabajo sobre la publicación de datos de pacientes.

Derecho a la privacidad y consentimiento informado. Los autores han obtenido el consentimiento informado de los pacientes y/o sujetos referidos en el artículo. Este documento obra en poder del autor de correspondencia. 


\section{Financiación}

\section{Ninguna.}

\section{Conflicto de intereses}

Los autores declaran no tener ningún conflicto de intereses.

\section{Bibliografía}

1. Nauta S, Deckers J, Akkerhuis K, et al. Short and lon g term mortality after myocardial infarction in patients with and without diabetes. Diabetes Care. 2012;35:2043-7.

2. Cubbon RM, Wheatcroft SB, Grant PJ, et al., Evaluation of Methods and Management of Acute Coronary Events Investigators. Temporal trends in mortality of patients with diabetes mellitus suffering acute myocardial infarction: A comparison of over 3000 patients between 1995 and 2003. Eur Heart J. 2007;28:540-5.

3. Jensen L, Moeng M, Thayssen P, et al. Influence of diabetes mellitus on clinical outcomes following primary percutaneus coronary intervention in patients with ST segment elevation myocardial infarction. Am J Cardiol. 2012;109:629-35.

4. Kralev S, Krause B, Papavassiliu T, et al. Clinical outcome of patients with diabetes presenting with ST elevation myocardial infarction and treated with concomitant use of glycoprotein IIb/IIla inhibitors. Cardiol J. 2009;16:234-40.

5. Donahoe SM, StewartGC, MC Cabe CH, et al. Diabetes and mortality following acute coronary syndromes. JAMA. 2007;298:765-75.

6. Authors/Task Force MembersRydén L, Grant PJ, Anker SD, et al. ESC Guidelines on diabetes, pre-diabetes, and cardiovascular diseases developed in collaboration with the EASD: the Task Force on diabetes, pre-diabetes, and cardiovascular diseases of the European Society of Cardiology (ESC) and developed in collaboration with the European Association for the Study of Diabetes (EASD). Eur Heart J. 2013;34:3035-87.

7. Krumholz HM, Anderson JL, Bachelder BL, Fesmire FM, Fihn SD, et al., Writing Committee Members. ACC/AHA 2008 performance measures for adults with ST-elevation and nonST-elevation myocardial infarction: A report of the American College of Cardiology/American Heart Association Task Force on Performance Measures (Writing Committee to develop performance measures for ST-elevation and non-ST-elevation myocardial infarction): Developed in collaboration with the American Academy of Family Physicians and the American College of Emergency Physicians: Endorsed by the American Association of Cardiovascular and Pulmonary Rehabilitation. Society for Cardiovascular Angiography and Interventions, and Society of Hospital Medicine. Circulation. 2008;118:2596-648.

8. De Luca G, Dirksen M, Spaulding C, et al. Impact of diabetes on long term outcome after primary angioplasty. Diabetes Care. 2013;36:1020-5.

9. De Luca G, Cassetti E, Marino P. Percutaneous coronary intervention-related time delay, patient's risk profile, and survival benefits of primary angioplasty vs lytic therapy in STsegment elevation myocardial infarction. Am J Emerg Med. 2009;27:712-9.
10. De Luca G, Biondi-Zoccai G, Marino P. Transferring patients with ST-segment elevation myocardial infarction for mechanical reperfusion: A meta-regression analysis of randomized trials. Ann Emerg Med. 2008;52:665-76.

11. Brener SJ, Mehran R, Dressler O, et al. Diabetes mellitus, myocardial infarction reperfusion and outcome in patients with acute ST elevation myocardial infarction treated with primary angioplasty (from HORIZONS AMI). Am J Cardiol. 2012;109:1111-6.

12. Van der Schaaf RJ, Henriques JPS, Wiersna JJ, et al. Primary percutaneus coronary intervention for patients with and without diabetes mellitus. Heart. 2006;92:117-8.

13. Keeley EC, Boura JA, Grines CL. Primary angioplasty versus intravenous thrombolytic therapy for acute myocardial infarction: A quantitative review of 23 randomised trials. Lancet. 2003;361:13-20.

14. Haffner SM, Lehto S, Ronnemaa T, et al. Mortality from coronary heart disease in subjects with type 2 diabetes and in nondiabetic subjects with and without prior myocardial infarction. N Engl J Med. 1998;339:229-34.

15. Timmer J, Ottervanger JP, Thomas $\mathrm{K}$, et al. Long term cause specific mortality after myocardial infarction in diabetes. EHJ. 2004;25:926-31.

16. Ishihara M, Kagawa E, Inoue I, et al. Impact of admission hyperglycemia and diabetes mellitus on short- and long-term mortality after acute myocardial infarction in the coronary intervention era. Am J Cardiol. 2007;99:1674-9.

17. Shechter M, Merz CN, Paul-Labrador MJ, et al. Blood glucose and platelet-dependent thrombosis in patients with coronary artery disease. J Am Coll Cardiol. 2000;35:300-7.

18. Khan M, Cubbon R, Mercer B, et al. Association of diabetes with increased all-cause mortality following primary percutaneous coronary intervention for ST-segment elevation myocardial infarction in the contemporary era. Diab Vasc Dis Res. 2012: 3-9.

19. Lekston A, Slonka G, Gasior M, et al. Comparison of early and long-term results of percutaneous coronary interventions in patients with ST elevation myocardial infarction, complicated or not by cardiogenic shock. Coron Artery Dis. 2010;21:13-9.

20. Gasior M, Pres D, Gierlotka M, et al. The influence of diabetes on in-hospital and long-term mortality in patients with myocardial infarction complicated by cardiogenic shock: results from the PL-ACS registry. Kardiol Pol. 2012;70:1215-24.

21. Grall S, Biere L, le Nezet M, et al. Relationship between betablocker and angiotensin-converting enzyme inhibitor dose and clinical outcome following acute myocardial infarction. Circ J. 2015;79:632-40.

22. Fuster V, Sanz G. A polypill for secondary prevention: Time to move from intellectual debate to action. Nat Clin Pract Cardiovasc Med. 2007;4:173.

23. Mukherjee D, Fang J, Chetcuti S, et al. Impact of combination evidence-based medical therapy on mortality in patients with acute coronary syndromes. Circulation. 2004;109:745-9.

24. Ho PM, Spertus JA, Masoudi FA, et al. Impact of medication therapy discontinuation on mortality after myocardial infarction. Arch Intern Med. 2006;166:1842-7.

25. Rasmussen JN, Chong A, Alter DA. Relationship between adherence to evidence-based pharmacotherapy and longterm mortality after acute myocardial infarction. JAMA. 2007;297:177-86. 